\title{
Quantitative contrast-enhanced ultrasound measurement of cerebrospinal fluid flow for the diagnosis of ventricular shunt malfunction
}

\author{
Robin Hartman, MS, ${ }^{1}$ Salavat Aglyamov, PhD, ${ }^{1}$ Douglas J. Fox Jr., MD, ${ }^{2}$ and \\ Stanislav Emelianov, PhD1 \\ 'Department of Biomedical Engineering, University of Texas at Austin; and 'NeuroTexas Institute, St. David's Hospital, \\ Austin, Texas
}

\begin{abstract}
OBJECT Cerebral shunt malfunction is common but often difficult to effectively diagnose. Current methods are invasive, involve ionizing radiation, and can be costly. The authors of this study investigated the feasibility of quantitatively measuring CSF flow in a shunt catheter using contrast-enhanced ultrasound.

METHODS A syringe pump was used to push a solution of gas-filled microbubbles at specific flow rates through a shunt catheter while a high-frequency ultrasound imaging system was used to collect ultrasound images for off-line processing. Displacement maps and velocity profiles were generated using a speckle-tracking method based on a cross-correlation algorithm. An additional correction factor, to account for a predictable underestimation and to adjust the measured flow rates, was calculated based on the geometry of the ultrasound imaging plane and assuming a simple model of laminar flow.
\end{abstract}

RESULTS The developed method was able to differentiate between physiologically relevant flow rates, including no flow and 0.006 to $0.09 \mathrm{ml} / \mathrm{min}$, with reasonable certainty. The quantitative measurement of flow rates through the catheter using this method was determined to be in good agreement with the expected flow rate.

CONCLUSIONS This study demonstrated that contrast-enhanced ultrasound has the potential to be used as a minimally invasive and cost-effective alternative method for outpatient shunt malfunction diagnosis.

http://thejns.org/doi/abs/10.3171/2014.12.JNS141014

KEY WORDS shunt malfunction; ultrasound; microbubbles; speckle tracking; hydrocephalus; CSF flow

$\mathrm{C}$ EREBRAL shunts (hereafter referred to as shunts) have been the standard treatment for hydrocephalus since the early 1960s. ${ }^{1}$ Despite the widespread use and effectiveness of shunts, malfunction and revision surgery are commonplace. The rate of malfunction of shunts in children is estimated to be $40 \%$ during the 1 st year following placement and $10 \%$ annually after the 1 st year. ${ }^{23}$ Common forms of shunt malfunction include occlusion, breakage, disconnection, migration, leakage, over-drainage, and infection. ${ }^{11}$

Current methods for diagnosing shunt malfunctions are often invasive, require ionizing radiation, or are expensive for the patient. Additionally, primary care physicians are increasingly being asked to examine patients for shunt malfunction and often rely on radiological imaging and results. These can be difficult to interpret without either baseline radiology reports or the expertise of a neurosurgeon. ${ }^{13}$ A method for noninvasively measuring CSF flow in a shunt or an easily interpreted method for checking flow to determine the need for further imaging is necessary. Alternative methods for CSF flow measurement in shunt systems have previously been proposed, including phasesensitive MRI, , 2,5,8,20 ultrasound imaging, $7,12,17,22,25$ and a device based on thermal convection. ${ }^{3,4,9,10,19}$

Ultrasound imaging is currently used in various clinical fields including cardiology, obstetrics, oncology, and pediatrics. Ultrasound systems are widely available and many are portable. In conventional ultrasound imaging, a high frequency ( $\mathrm{MHz}$ range) pulse is transmitted into tissue, and waves backscattered from structures and mechanical inhomogeneities within tissue are recorded to form an image. Ultrasound is a nonionizing and noninvasive imaging modality. In contrast-enhanced ultrasound, micrometersized bubbles of gas, which are characterized by a very high acoustic impedance mismatch relative to soft tissue, create a strong ultrasound backscatter signal. 
Previously, Doppler ultrasound imaging has been proposed as a method for measuring CSF flow rates in hydrocephalus shunts with and without added contrast agents. ${ }^{17,22}$ Researchers developed an imaging system that could detect lower flow rates than previously measured using Doppler alone by generating microbubbles in the shunt using low frequency ultrasound. The velocity of the bubbles was then measured by Doppler ultrasound using a single element transducer. Doppler ultrasound, however, has limited sensitivity to low flow rates, which have peak velocities below a few millimeters per second.

In this article, we discuss the utility of contrast-enhanced ultrasound as a safe, minimally invasive, nonionizing, reliable, and cost-effective method for quantitative measurement of CSF flow. We investigated a method based on contrast-enhanced ultrasound imaging with a linear array transducer and a 2D speckle-tracking algorithm to quantitatively measure CSF flow in a shunt system. This method is sensitive to very low flow rates, simple to implement, cost-effective, and could be performed in outpatient settings using widely available clinical ultrasound systems. To assess CSF flow, microbubbles are injected into the shunt system and then imaged using an array transducer. Injection of the microbubbles into the shunt reservoir carries little to no risk of infection if performed following the same conditions and procedures as a shunt tap. ${ }^{24}$ Motion estimation (e.g., 2D cross-correlation speckle tracking) is performed using a sequence of ultrasound frames, and flow velocity distribution in the shunt is determined from the measured displacement vectors. Here we conducted a study to establish the feasibility of quantitative measurement of flow using contrast-enhanced ultrasound.

\section{Methods}

A Medtronic ventricular shunt catheter was placed in the flow-mimicking setup shown in Fig. 1 upper. A syringe infusion pump was used to flow a dilute solution of ultrasound contrast agent through the shunt catheter. Ultrasound images were collected using the Vevo2100 ultrasound imaging system (VisualSonics Inc.). The images were then saved for offline processing. Specifically, a 2D cross-correlation algorithm was used to determine displacement and thus a 2D flow velocity profile in the shunt catheter. Subsequently, the 2D velocity profile was used to calculate flow rate, assuming laminar flow in the catheter. The width of the ultrasound beam, comparable in size to the inner diameter of the catheter, produced a predictable underestimation of the flow rate. Therefore, the flow rates calculated from the velocity profile were adjusted by a predetermined correction factor. Further details of imaging experiments, flow rate measurements, and algorithms are described below.

\section{Experimental Setup}

The flow simulation setup is shown in Fig. 1 upper. A Medtronic shunt catheter with an inner diameter of $1.2 \mathrm{~mm}$ and outer diameter of $2.4 \mathrm{~mm}$ was suspended in a container filled with degassed water. The bottom of the container was lined with 6.5 -mm-thick acoustic foam to reduce acoustic reflections. The catheter was placed parallel to the $\mathrm{x}$-axis of the imaging plane at the geometric focus of the
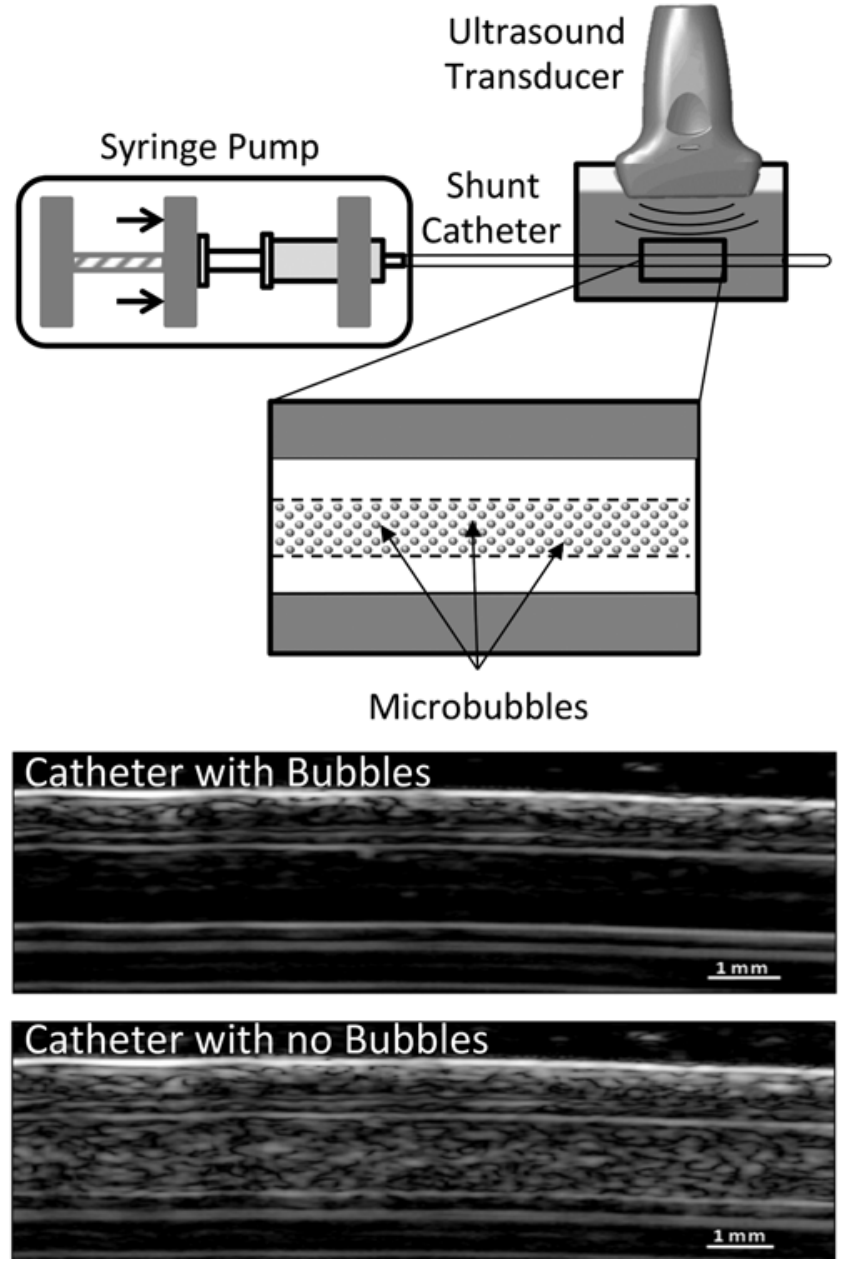

FIG. 1. Upper: Experimental setup consisting of a syringe pump to simulate flow in a shunt catheter and an ultrasound transducer for image acquisition. Lower: Representative B-mode ultrasound images of the ventricular shunt catheter with and without microbubbles (ultrasound contrast agent).

transducer on the z-axis (axes shown in Fig. 2). Positioning in the $y$-axis was achieved by adjusting the position of the transducer relative to the catheter using a manual motion stage. A 5-ml syringe with a diluted microbubble solution was placed in a syringe infusion pump (Fusion 400, Chemyx). Micromarker nontargeted contrast agent (VisualSonics Inc.) was reconstituted with $0.7 \mathrm{ml}$ of saline solution and diluted to $0.01 \%$ of the original concentration with deionized water (approximately $2 \times 10^{5}$ microbubbles/ $\mathrm{ml})$. The microbubbles were lipid-coated, contained a gas mixture of nitrogen and perfluorobutane, and had a median diameter between 2.3 and $2.9 \mu \mathrm{m}$. The same microbubble solution was used for the duration of the experiment (approximately 2 hours); however, the syringe did need to be shaken occasionally to redistribute the microbubbles. The syringe pump was programmed for 0.00 (off or no flow), $0.01,0.02,0.05$, and $0.10 \mathrm{ml} / \mathrm{min}$ to mimic physiological CSF flow rates..$^{14}$ Figure 1 lower presents B-mode ultrasound images of the shunt before and after bubble introduction. The images were obtained using a $15-\mathrm{MHz}$ ultrasound transducer (MS200, VisualSonics Inc.). 


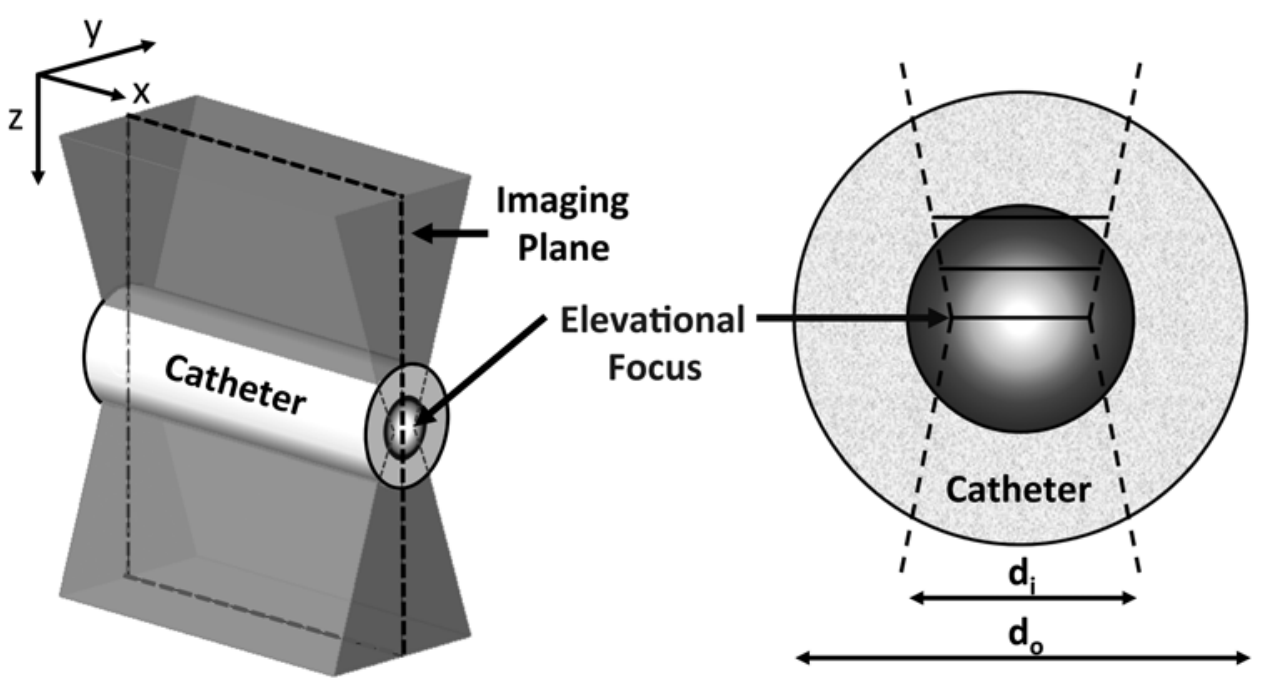

FIG. 2. A schematic representation of the ultrasound beam in the axial $(\mathrm{z})$, lateral $(\mathrm{x})$, and elevational $(\mathrm{y})$ directions. The width of the ultrasound beam in the elevational direction is on the order of the inner diameter $\left(\mathrm{d}_{\mathrm{i}}\right)$ of the catheter (left). The variations in the elevational width of the ultrasound beam results in an underestimation of velocity. The effect of the elevational beam width on the measured flow velocity can be accounted for by calculating the mean velocity across the elevational width of the beam in a simple laminar flow model (right). $d_{0}=$ outer diameter.

To evaluate contrast-enhanced ultrasound flow rate measurement in an ex vivo model, a proximal shunt catheter was inserted subcutaneously in a skin-on pork-belly tissue sample. For this experiment, the same setup shown in Fig. 1 upper was used, except that a shunt valve (PS Medical CSF-Flow Control Valve, Medtronic) was connected between the syringe and the catheter. The tissue sample was positioned under the transducer, following the same procedure as the catheter in water study mentioned previously. The microbubbles were delivered via a $50-\mu 1$ bolus injection directly into the reservoir with a 25 -gauge needle, and images were acquired once the microbubbles reached the field of view of the imaging transducer. Ultrasound coupling gel (Parker Laboratories) was used for acoustic coupling of the ultrasound transducer and the tissue sample during the imaging. A 21-MHz ultrasound transducer (MS250, VisualSonics Inc.) was used for this study, and images were acquired for 2 syringe pump settings: 0.05 and $0.1 \mathrm{ml} / \mathrm{min}$.

\section{Syringe Pump Flow Calibration}

For low flow rates, flow output of the syringe infusion pump depends on the height of the pump relative to the outlet. To determine the actual flow rates in our experimental setup, the syringe pump was run using a waterfilled syringe (Fig. 1), and the catheter was positioned such

TABLE 1. Syringe pump flow calibration results*

\begin{tabular}{cc}
\hline Syringe Pump Setting & Mean Calibration Flow Rate (SD) \\
\hline 0.00 & $-0.002(0.0012)$ \\
\hline 0.01 & $0.006(0.0008)$ \\
\hline 0.02 & $0.014(0.0018)$ \\
\hline 0.05 & $0.043(0.0031)$ \\
\hline 0.10 & $0.090(0.0041)$ \\
\hline
\end{tabular}

${ }^{*}$ All values are $\mathrm{ml} / \mathrm{min}$. that the tip dripped into a vial positioned on a microbalance (Mettler Toledo, LLC). The weight change due to water in the vial was used to determine the actual volume of water dispensed by the syringe pump after 15 minutes. The mean of 10 measurements at each flow rate was used to determine the actual flow rates. These true flow rates were used to determine the accuracy of the flow rates measured using contrast-enhanced ultrasound. The results of the calibration are shown in Table 1.

\section{Imaging System}

Images of the shunt catheter were acquired with a Vevo 2100 ultrasound system. The system was equipped with both a $15-\mathrm{MHz}$ linear array transducer and a 21-MHz linear array transducer. The 3D geometry of the transducer ultrasound beam is presented in Fig. 2 left, where 3 directions are shown: along the ultrasound beam (axial, z-axis), orthogonal to the ultrasound beam (lateral, $x$-axis), and out of the imaging plane (elevational, y-axis). Before acquisition of each image set, the pump was allowed to run for 5 seconds to ensure consistent flow.

To image the shunt catheter in a water container, a 15$\mathrm{MHz}$ transducer was used with transmit power set to 5\% to reduce acoustic force and to prevent the bubbles from being pushed to the bottom of the catheter. Ultrasound images were collected at 70 frames per second, with the center of the catheter positioned at a depth of $18 \mathrm{~mm}$ (the geometric focus of the $15-\mathrm{MHz}$ transducer), and maximum imaging depth was set to $22 \mathrm{~mm}$. The images were $17 \mathrm{~mm}$ wide in the lateral direction with 16 A-lines per millimeter. Five independent image sets were acquired for each of the 5 programmed flow rates. In the ex vivo experiment, a 21-MHz linear array transducer was used with transmit power set to $5 \%$. Images were collected at 25 frames per second, with the center of the catheter positioned at a depth of $15 \mathrm{~mm}$ (the geometric focus of the 21-MHz transducer) and maximum imaging depth set to 
$30 \mathrm{~mm}$. The images were $23 \mathrm{~mm}$ wide with about $22 \mathrm{~A}-$ lines per millimeter.

\section{Speckle-Tracking Method Based on Cross-Correlation for Velocity Measurement}

A speckle-tracking algorithm based on a phase-sensitive cross-correlation method was used in our studies..$^{18}$ To compute the displacement images, a Hamming-weighted $0.81 \times 0.17 \mathrm{~mm}$ (lateral by axial) kernel was used for the $15-\mathrm{MHz}$ images, and a $1.13 \times 0.16 \mathrm{~mm}$ kernel was used for the $21-\mathrm{MHz}$ images. The search area was limited to 3 times the expected maximum pixel displacement for a particular flow rate..$^{18}$ The correlation was performed on 31 consecutive frames spanning 0.42 seconds for the catheter in water study and 1.2 seconds for the ex vivo study. To improve the quality of the lateral velocity measurements, the autocorrelation compensation method was used, in which the displacement result of the autocorrelation of the first image was subtracted from the displacement result of the cross-correlation of the first and second images. ${ }^{15}$ This approach, described in detail elsewhere,,$^{15}$ demonstrated significant noise reduction and improvement of the motion estimation in the lateral direction.

\section{Flow Correction Factor}

Due to the finite focal width of the ultrasound imaging plane in the elevational direction (Fig. 2) and the small diameter of the catheter, the velocity of the speckle motion in an ultrasound image is actually the mean velocity of all of the bubbles within the catheter. To take into account this systematic error, we introduce a correction factor based on the ultrasound beam size in the elevational direction and the inner diameter of the catheter. The $15-\mathrm{MHz}$ and $21-$ $\mathrm{MHz}$ transducers have a beam thickness of 0.770 and 0.765 $\mathrm{mm}$ at their respective geometric foci, distances spanning more than half of the inner diameter of the catheter $(1.2$ $\mathrm{mm})$. To determine the velocity profile, a simple model of the beam profile was implemented together with an assumption of laminar flow. The velocities within the catheter were averaged across the beam width, as shown in Fig. 2. The expected flow rate for an input flow rate was then calculated as the integral of the expected velocity profile over the cross-sectional area of the catheter. The input flow rate was divided by the expected flow rate to obtain a correction factor that we used to adjust our measured flow rates. For the 15-MHz transducer, this correction factor was estimated as 1.44 at the geometric focus of the transducer. For the 21-MHz transducer used in the ex vivo experiment, the correction factor was 1.49 at the geometric focus.

\section{Analysis}

Results of the cross-correlation speckle-tracking algorithm were visualized using Matlab (MathWorks, Inc.). The total displacement over 30 correlation frames was calculated as the pixel-wise sum of the displacement vectors frame to frame and was used to create a velocity map. A region of interest was selected by identifying the inner walls of the catheter on the ultrasound images and was applied, as a mask, to the velocity map. Velocity profiles were evaluated by calculating the mean velocity along each lateral line of the masked velocity map. Standard deviations were calculated for all the velocities at each vertical position within the catheter. Profiles from each of the independent image sets were averaged to obtain a mean velocity profile for each flow rate. Standard deviations of the mean velocity at each vertical position for all the image sets were also calculated. The flow rate in the catheter was calculated from the velocity profile, and the results were compared with the flow rates obtained in the calibration experiment.

\section{Results}

As can be seen in the velocity maps shown in Fig. 3, the speckle-tracking method was able to differentiate between high and low flow rates within the expected physiological range. At high flow rates, the flow is laminar. At lower flow rates such as 0.006 and $0.014 \mathrm{ml} / \mathrm{min}$, there is lower flow in the upper portion of the catheter and higher flow in the lower portion, probably due to a combination of bubble buoyancy and bubble adhesion to the wall of the catheter. It is important to note that this method is able to detect lateral motion, a limitation of Doppler ultrasound. In patients, catheters will follow a less direct and predictable path; thus, sensitivity to both axial and lateral motion is important.

The 5 experimental flow rates can be easily differentiated from one another based on the velocity profiles as shown in Fig. 4. Additionally, the velocity measurements at each flow rate do not vary significantly, indicating consistency in our method. The measured velocity profiles show a slight broadening and smaller peak velocity compared with the expected profiles. This could be attributed

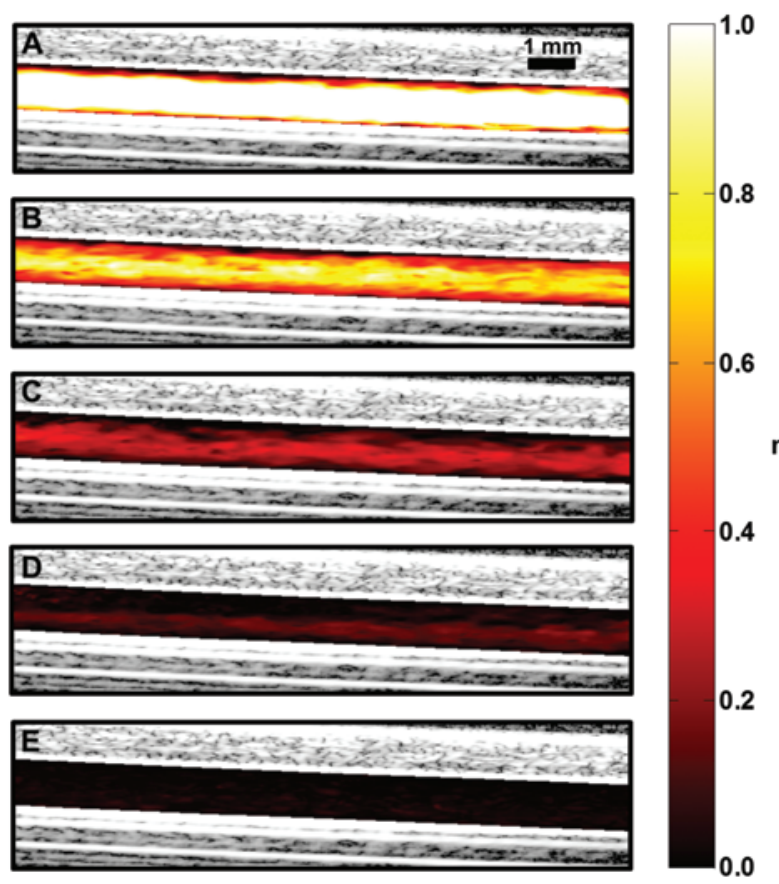

FIG. 3. Velocity maps for flow rates of $0.090 \mathrm{ml} / \mathrm{min}(\mathrm{A}), 0.043 \mathrm{ml} / \mathrm{min}$ (B), $0.014 \mathrm{ml} / \mathrm{min}$ (C), $0.006 \mathrm{ml} / \mathrm{min}$ (D), and $0.000 \mathrm{ml} / \mathrm{min}(\mathrm{E})$ in a shunt catheter in water. Prior to display, a region of interest was defined based on the B-mode images such that errant velocity measurements outside the catheter are not shown. 
to a number of factors including a slight change in fluid viscosity with the addition of the bubbles or error due to the catheter having a slight curve out of plane, which was not taken into account when averaging the displacement values over the length of the catheter.

Final measured flow rates were compared with the actual flow rates and are shown in Fig. 5. Measured flow rates were adjusted by a correction factor of 1.44 for the $15-\mathrm{MHz}$ transducer and 1.49 for the $21-\mathrm{MHz}$ transducer.

Flow rate measurement in an ex vivo tissue sample produced results consistent with the previous studies (Fig. 6). At $0.09 \mathrm{ml} / \mathrm{min}$, the measured flow rate was $0.098 \mathrm{ml} / \mathrm{min}$, and at $0.043 \mathrm{ml} / \mathrm{min}$, the flow rate was $0.04 \mathrm{ml} / \mathrm{min}$. These values fall within the expected range of error shown in Fig. 5.

\section{Discussion}

Shunt systems have been the standard of treatment for hydrocephalus for several decades, but they are still exceedingly prone to malfunction. Blockage, breakage, infection, migration, over-drainage, and infiltration by tissue are all common problems with shunt systems, most of
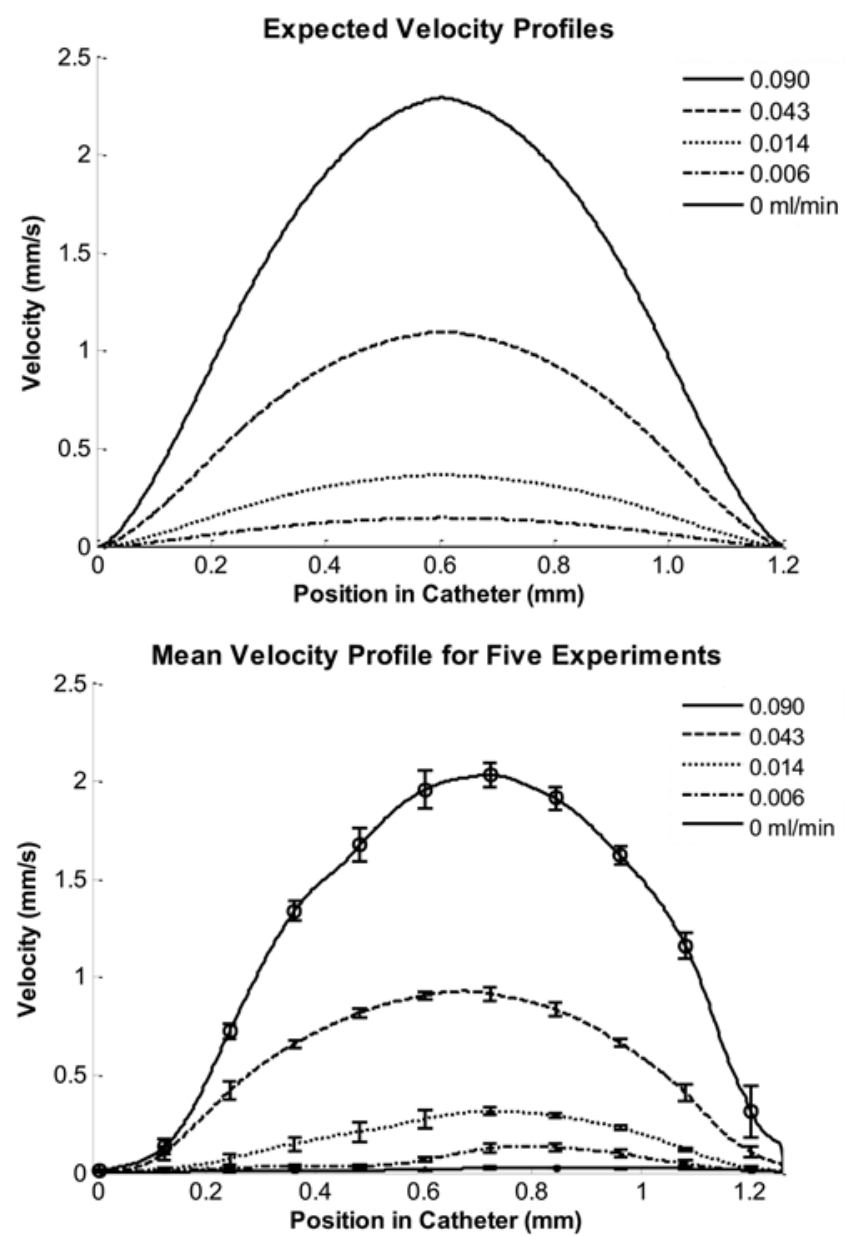

FIG. 4. Upper: Expected velocity profiles based on modeled data where the transducer beam width was taken into account. Lower: Mean velocity profiles obtained from 5 independent measurements. Error bars indicate the standard deviation, at that particular position, of 5 independent velocity profiles.

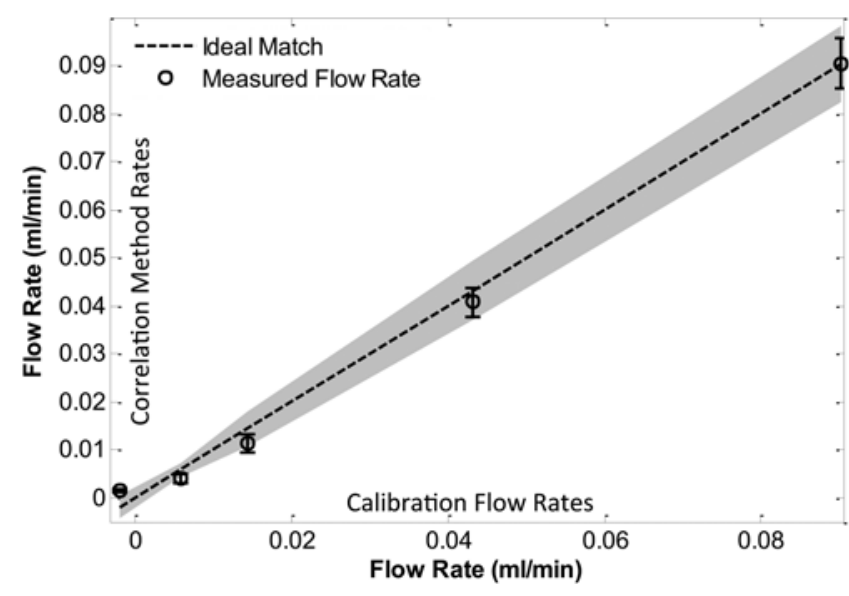

FIG. 5. Measured flow rate versus true flow rate (as determined by flow calibration experiments). Gray region represents standard deviation of flow calibration results. Error bars represent standard deviations of measured data.

which are not easy to diagnose with certainty. There have been several proposed devices and methods for detecting flow in a shunt system, but none have been sensitive enough to quantify flow while remaining cost-effective and safe for infants and children.

A standard ventricular shunt tap may give an initial indication of the type of malfunction such as whether the proximal catheter is obstructed; however, the literature suggests that the shunt tap may be misleading in cases where there is good flow upon tapping of the shunt. This method is also not quantitative. Although the opening pressure could be estimated by the height of a column of CSF, this would not be as sensitive as the method proposed here..$^{6,21}$

MR phase imaging is typically sensitive to flow rates of $0.03 \mathrm{ml} / \mathrm{min}$ and higher,, 5 and in some cases has shown sensitivity to flow rates as low as $0.013 \mathrm{ml} / \mathrm{min} .{ }^{8}$ Though MRI shows sensitivity to fairly low flow rates, it requires that the patient remain still for the entire imaging session, which can be difficult for young children. Additionally, it can be expensive and is not widely available outside of hospitals. MRI is very useful as a whole-body imaging method for identifying breaks, blockages, and other mechanical defects within the entire shunt system, and ultrasound imaging could be used to determine if there is a further need for MRI.

The ShuntCheck device, another method for measuring flow in a shunt, is extremely simple, employing thermal convection to determine if there is flow or no flow in a portion of the shunt catheter. While it has demonstrated some specificity, a binary flow or no flow determination, it is not sensitive to flow rates below $0.08 \mathrm{ml} / \mathrm{min},{ }^{4}$ and it is not inherently quantitative as it relies simply on the speed of temperature change, which could be affected by external factors. ${ }^{8}$

Doppler ultrasound has also been previously proposed for shunt flow detection. CSF is acoustically transparent since it has no intrinsic reflector, but one study found that debris in the CSF was enough to produce a satisfactory Doppler signal and flow rates between 2.3 and $4.6 \mathrm{ml} / \mathrm{min}$ were detected. ${ }^{22}$ The authors also found that in CSF with 


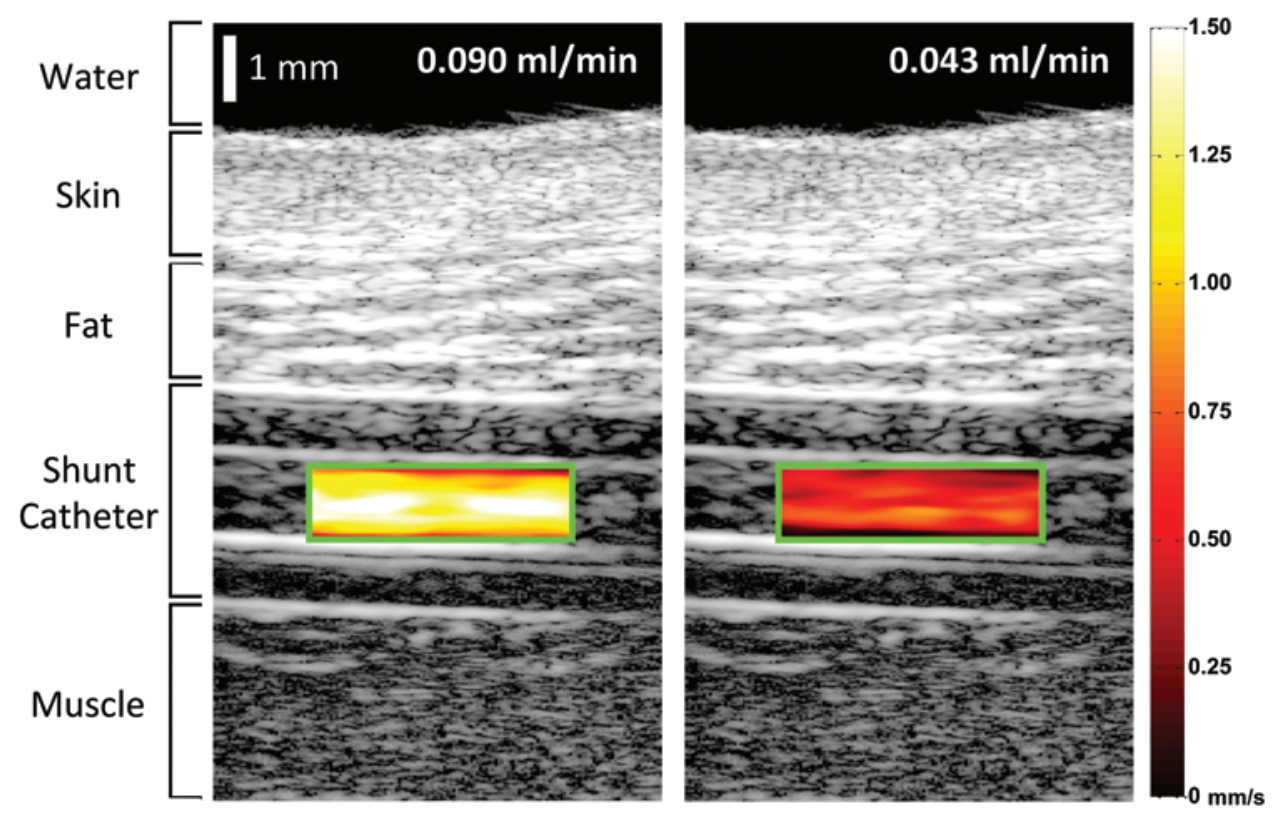

FIG. 6. Ex vivo B-mode images of a skin-on pork-belly sample with shunt catheter subcutaneously implanted. Velocity maps within the catheter at input flow rates of $0.09 \mathrm{ml} / \mathrm{min}$ (left) and $0.043 \mathrm{ml} / \mathrm{min}$ (right) are shown overlaid on the B-mode images. Measured flow rates, calculated from the velocity maps, were $0.098 \mathrm{ml} / \mathrm{min}$ and $0.040 \mathrm{ml} / \mathrm{min}$, respectively.

no source of contrast, turbulent flow at junction points in the shunt system could sometimes produce bubbles for contrast. In several cases, it could not be determined if a lack of flow was indicative of malfunction or if there was simply no contrast in the CSF. This method is also not sensitive to low flow rates that could occur in a functioning shunt. Another group of researchers employed Doppler for flow detection and introduced contrast by the acoustic generation of microbubbles within a shunt catheter, eliminating the need for intrinsic contrast and making the method noninvasive. ${ }^{17}$ However, Doppler ultrasound is angle-dependent, and thus requires knowledge of the 3D shape of the shunt catheter. The authors showed an overestimation at lower velocities (on the order of the higher velocities demonstrated here) and explained that the overestimation is due to buoyancy. ${ }^{17}$ The speed of the bubble upwards adds additional motion toward the transducer in the axial direction, resulting in an overestimation of the peak and mean velocities. Additionally, Doppler ultrasound still requires a fairly high flow rate of $0.03 \mathrm{ml} / \mathrm{min}$ for flow detection, like MRI. ${ }^{17}$

Our results indicate a higher sensitivity than reported by others, ${ }^{17}$ who were able to demonstrate detection as low as $0.05 \mathrm{ml} / \mathrm{min}$. As shown in Fig. 5, our results show a very slight underestimation at lower flow rates $(0.006$ and $0.014 \mathrm{ml} / \mathrm{min}$ ) and good agreement at the higher flow rates $(0.043$ and $0.09 \mathrm{ml} / \mathrm{min})$. Our method does not suffer from the same directional error as Doppler, where microbubble buoyancy adds velocity in the axial direction and contributes to overestimation at low flow rates, because we employ information in 2 dimensions. Our underestimation could be a result of adhesion of the bubbles to the catheter in combination with buoyancy forcing bubbles into the upper portion of the catheter. This can be seen in the velocity profiles at 0.006 and $0.014 \mathrm{ml} / \mathrm{min}$. The profiles for these flow rates are not symmetric parabolas. The left side (upper portion of the catheter) is significantly smaller and flat- ter than the right side (lower portion of the catheter). This error could be minimized by increasing the transmission power, to further counteract the buoyancy of the bubbles at lower flow rates, or by reducing the bubble concentration.

In our method, the elevational beam width of the transducer and the inner diameter of the catheter need to be taken into account to determine an accurate expected velocity profile and flow rate. Since the beam profile changes with depth, a better model for when the beam is wider than the transducer would need to be developed for imaging outside of the geometric focus of the transducer. Once these factors are accounted for, our method shows strong agreement with the true flow rate. This strong agreement coupled with the use of only 30 frames, or 0.42 seconds of data, for the $15-\mathrm{MHz}$ transducer also indicates that this would be a very robust and rapid method for flow measurement.

In the future, this method will be developed further such that kernel size and search area can be selected automatically based on initial measurements, and the operator would simply select a region of interest to be used for flow rate calculation. The lowest detectable flow rate using contrast-enhanced ultrasound $-0.006 \mathrm{ml} / \mathrm{min}$ in our studiesis mainly defined by the characteristics of the ultrasound system. It may be possible to increase sensitivity to lower flow rates by modifying properties of the ultrasound system or the correlation parameters used in flow rate measurement. The sensitivity necessary for differentiating between a functioning and malfunctioning shunt should be determined in future clinical studies. Finally, rather than injecting bubbles directly into the shunt system, microbubbles could be generated externally through cold cavitation, although the safety and consistency of this method would need to be explored further. ${ }^{16,17}$

The proposed method permits not only detection of flow but also provides quantitative information about flow rate that could aid in singular and longitudinal studies of 
fluid mechanics of shunts. Studying the changes in CSF flow rate in a shunt in relation to external factors could give additional valuable information about hydrocephalus.

\section{Conclusions}

We have demonstrated the validity and feasibility of our proposed method to measure CSF flow in shunts using contrast-enhanced ultrasound and a cross-correlationbased speckle-tracking algorithm. The method is able to differentiate between no flow and flow rates between $0.006 \mathrm{ml} / \mathrm{min}$ and $0.090 \mathrm{ml} / \mathrm{min}$, which are within the physiological range of CSF flow in shunts. The results also agree reasonably well with the expected flow rates based on the experimental setup. In our studies, we used a commercially available ultrasound system. This method could easily be translated to a clinical ultrasound system and setting, potentially reducing the need for ionizing radiation-based imaging methods, while also allowing for convenient imaging and measurement of CSF flow using a portable imaging system. It could also be used in the emergency room to quickly evaluate the need for further evaluation of the shunt system using MRI. Finally, measurement of CSF flow in a patient over time could provide valuable information about CSF flow dynamics in hydrocephalus patients with implanted shunts.

\section{References}

1. Aschoff A, Kremer P, Hashemi B, Kunze S: The scientific history of hydrocephalus and its treatment. Neurosurg Rev 22:67-95, 1999

2. Chang HS: Detection of CSF flow in the ventriculo-peritoneal shunt using MRI. J Comput Assist Tomogr 20:429-433, 1996

3. Chiba Y, Ishiwata Y, Suzuki N, Muramoto M, Kunimi Y: Thermosensitive determination of obstructed sites in ventriculoperitoneal shunts. J Neurosurg 62:363-366, 1985

4. Chiba Y, Yuda K: Thermosensitive determination of CSF shunt patency with a pair of small disc thermistors. J Neurosurg 52:700-704, 1980

5. Drake JM, Martin AJ, Henkleman RM: Determination of cerebrospinal fluid shunt obstruction with magnetic resonance phase imaging. J Neurosurg 75:535-540, 1991

6. Duhaime AC, Wiley JF II: Ventricular shunt and burr hole puncture, in King C, Henretig FM (eds): Textbook of Pediatric Emergency Procedures, ed 2. Philadelphia: Lippincott Williams \& Wilkins, 2008, pp 515-521

7. Flitter MA, Buchheit WA, Murtagh F, Lapayowker MS: Ultrasound determination of cerebrospinal fluid shunt patency. Technical note. J Neurosurg 42:728-730, 1975

8. Frank E, Buonocore M, Hein L: Magnetic resonance imaging analysis of extremely slow flow in a model shunt system. Childs Nerv Syst 8:73-75, 1992

9. Go KG, Melchior HJ, Lakke JPWF: A thermosensitive device for the evaluation of the patency of ventriculo-atrial shunts in hydrocephalus. Acta Neurochir (Wien) 19:209-216, 1968

10. Go KG, van der Veen PH, van den Berg JW: Detection of CSF flow in ventriculo-atrial shunts by cold transfer. Dev Med Child Neurol 12 (Suppl 22): 69-72, 1970

11. Goeser CD, McLeary MS, Young LW: Diagnostic imaging of ventriculoperitoneal shunt malfunctions and complications. Radiographics 18:635-651, 1998

12. Hara M, Kadowaki C, Konishi Y, Ogashiwa M, Numoto M, Takeuchi K: A new method for measuring cerebrospinal fluid flow in shunts. J Neurosurg 58:557-561, 1983
13. Iskandar BJ, McLaughlin C, Mapstone TB, Grabb PA, Oakes WJ: Pitfalls in the diagnosis of ventricular shunt dysfunction: radiology reports and ventricular size. Pediatrics 101:10311036, 1998

14. Kadowaki C, Hara M, Numoto M, Takeuchi K: Factors affecting cerebrospinal fluid flow in a shunt. Br J Neurosurg 1:467-475, 1987

15. Kim S, Aglyamov SR, Park S, O’Donnell M, Emelianov SY: An autocorrelation-based method for improvement of subpixel displacement estimation in ultrasound strain imaging. IEEE Trans Ultrason Ferroelectr Freq Control 58:838843,2011

16. Lam KW, Drake JM, Cobbold RSC: Generation and maintenance of bubbles in small tubes by low-frequency ultrasound. J Acoust Soc Am 106:3719-3729, 1999

17. Lam KW, Drake JM, Cobbold RSC: Noninvasive cerebrospinal fluid shunt flow measurement by Doppler ultrasound using ultrasonically excited bubbles: a feasibility study. Ultrasound Med Biol 25:371-389, 1999

18. Lubinski MA, Emelianov SY, O’Donnell M: Speckle tracking methods for ultrasonic elasticity imaging using shorttime correlation. IEEE Trans Ultrason Ferroelectr Freq Control 46:82-96, 1999

19. Madsen JR, Abazi GS, Fleming L, Proctor M, Grondin R, Magge S, et al: Evaluation of the ShuntCheck noninvasive thermal technique for shunt flow detection in hydrocephalic patients. Neurosurgery 68:198-205, 2011

20. Martin AJ, Drake JM, Lemaire C, Henkelman RM: Cerebrospinal fluid shunts: flow measurements with MR imaging. Radiology 173:243-247, 1989

21. Rocque BG, Lapsiwala S, Iskandar BJ: Ventricular shunt tap as a predictor of proximal shunt malfunction in children: a prospective study. J Neurosurg Pediatr 1:439-443, 2008

22. Sgouros S, John P, Walsh AR, Hockley AD: The value of colour Doppler imaging in assessing flow through ventriculoperitoneal shunts. Childs Nerv Syst 12:454-459, 1996

23. Sood S, Ham SD, Canady AI: Current treatment of hydrocephalus. Neurosurg Q 11:36-44, 2001

24. Spiegelman L, Asija R, Da Silva SL, Krieger MD, McComb JG: What is the risk of infecting a cerebrospinal fluid-diverting shunt with percutaneous tapping? J Neurosurg Pediatr 14:336-339, 2014

25. Widder DJ, Davis KR, Taveras JM: Assessment of ventricular shunt patency by sonography: a new noninvasive test. AJR Am J Roentgenol 147:353-356, 1986

\section{Author Contributions}

Conception and design: all authors. Acquisition of data: Hartman. Analysis and interpretation of data: Hartman. Drafting the article: Hartman. Critically revising the article: Emelianov, Aglyamov, Fox. Reviewed submitted version of manuscript: all authors. Approved the final version of the manuscript on behalf of all authors: Emelianov. Statistical analysis: Hartman. Study supervision: Emelianov, Aglyamov.

\section{Supplemental Information}

\section{Previous Presentation}

Portions of this work were presented in poster form at the 2013 Biomedical Engineering Society (BMES) Annual Meeting (September 27, 2013) in Seattle, Washington, and in oral presentation form at the 2014 BMES Annual Meeting (October 25, 2014) in San Antonio, Texas.

\section{Correspondence}

Stanislav Emelianov, Department of Biomedical Engineering, University of Texas at Austin, 107 W. Dean Keeton, BME Bldg., Austin, TX 78712. email: emelian@mail.utexas.edu. 